\title{
Prospecção de Tecnologias para Telerreabilitação: inovação nos atendimentos fisioterapêuticos
}

\author{
Prospecting Technologies for Telerehabilitation: innovation in \\ physiotherapeutic care
}

\author{
Marinêz Boeing Ruaro ${ }^{1}$ \\ João Afonso Ruaro ${ }^{1}$ \\ Daniel de Paula ${ }^{1}$ \\ ${ }^{1}$ Universidade Estadual do Centro-Oeste, Campus Cedeteg, Guarapuava, PR, Brasil
}

\begin{abstract}
Resumo
A pandemia da Covid-19 causou uma demanda expressiva pela telerreabilitação fisioterapêutica - uma modalidade de telessaúde - em especial pelo isolamento social e pelo elevado número de pacientes com sequelas da síndrome pós-Covid. Assim, as Tecnologias da Informação e Comunicação (TICs) surgem como uma alternativa ao tratamento convencional. Este estudo objetivou demonstrar o panorama das principais TICs e como estão sendo usadas na telerreabilitação, por meio de busca patentária (Orbit Intelligence ${ }^{\circledR}$ ) e prospecção bibliométrica (Pubmed, PEDro e Cochrane). Os resultados mostraram tecnologias e dispositivos (smartphones, aplicativos) que têm viabilizado diversas formas de telerreabilitação, que servem como ferramenta para que os profissionais da saúde possam continuar a assistência. Além disso, demonstram que a produção científica (12.660 artigos) é muito superior à tecnológica (89 patentes), sinalizando inadequação da transferência de tecnologia. Conclui-se que há TICs, ferramentas e dispositivos suficientes para oferecer a telerreabilitação de maneira adequada, acessível e com potencial para desenvolvimento de inovações.
\end{abstract}

Palavras-chave: Telessaúde. Fisioterapia. TICs.

\begin{abstract}
The Covid-19 pandemic caused an expressive demand for physical therapy telerehabilitation - a telehealth modality - especially due to social isolation and the high number of patients with post-Covid syndrome sequelae. Therefore, Information and Communication Technologies (ICTs) emerge as an alternative to conventional treatment. This study aimed to demonstrate an overview of the main ICTs and how they are being used in telerehabilitation, through patent search (Orbit Intelligence ${ }^{\circledR}$ ) and bibliometric prospecting (Pubmed, PEDro and Cochrane). The results showed technologies and devices (smartphones, applications) that have enabled various forms of telerehabilitation, which serve as a tool for health professionals to continue providing care. Furthermore, they demonstrate that a scientific production (12.660 articles) is superior to a technological production (89 patents), evidencing inadequacy of technology transfer. It is concluded that there are enough ICTs, tools and devices to offer telerehabilitation in an adequate, accessible way and with potential for the development of innovations.
\end{abstract}

Keywords: Telehealth. Physiotherapy. ICTs

Áreas Tecnológicas: Prospecção Tecnológica. Telessaúde. 


\section{Introdução}

A pandemia da Covid-19 foi decretada em 11 de março de 2020 pela Organização Mundial da Saúde (OMS) e com ela o mundo teve que enfrentar não só uma doença nova, mas também situações inusitadas, que exigiram mudanças radicais de comportamento, nos níveis individual e comunitário (OLIVEIRA et al., 2020). Esse acontecimento alterou os hábitos de toda população, inclusive dos serviços de saúde, por serem possíveis fontes de contágio. Isso direcionou a atenção a novos modelos de atendimento eletivo, os quais evitam o contato direto entre o profissional da saúde e o cliente, resultando em uma expansão sem precedentes na telessaúde em todo o mundo (GREENHALGH; KOH; CAR, 2020).

Telessaúde é um conceito amplo que engloba a prestação de serviços de atenção à saúde em seus diferentes níveis (primário, secundário e terciário) de maneira remota, com o apoio das Tecnologias da Informação e Comunicação (TICs), visando à interação entre profissionais de saúde ou entre estes e seus pacientes (BRASIL, 2016). As TICs, por sua vez, tornaram-se uma opção valiosa também para a reabilitação, apoiando o nascimento de um novo ramo da telessaúde, a telerreabilitação. Esta envolve a entrega remota de diferentes serviços de reabilitação por meio de tecnologias de telecomunicações, otimizando o acesso aos serviços (BONNEVIE et al., 2021).

Como parte da equipe de reabilitação, o fisioterapeuta passou a experimentar um processo disruptivo na sua assistência tradicional com o início da pandemia, sendo identificada a necessidade de implementar medidas e recursos para manter a assistência profissional de forma não presencial aos usuários. Nesse caso, as TICs oferecem possibilidades oportunas e o domínio da sua utilização passa a ser primordial aos profissionais que desejam se destacar no mercado (NIC; CETIC, 2020).

A combinação entre reabilitação e desenvolvimento tecnológico tem se mostrado essencial para a entrega de assistência fisioterapêutica a distância frente à pandemia, pois, assim, foi possível dar continuidade ao tratamento, mesmo quando se exigiram medidas protetivas mais severas com suspenção temporária de alguns serviços de saúde de maneira presencial (FIORATTI et al., 2020).

A telerreabilitação permite a assistência a um maior número de pessoas, mesmo com número reduzido de profissionais, e minimiza a sobrecarga de assistência presencial nos serviços de saúde; não necessita aguardar o retorno da assistência eletiva à saúde, extingue o risco de contágio e disseminação da Covid-19 e viabiliza um serviço sem a necessidade do deslocamento dos pacientes, permitindo, assim, uma maior segurança a todos (GONZALEZ-GEREZ et al., 2020). Assim, esse modelo auxilia a suprir o aumento da demanda, gerada pela necessidade de assistência a pacientes com síndrome pós-Covid-19, já que diversos sintomas podem persistir por longos períodos após o fim da fase aguda da doença (KARSTEN; MATTE; ANDRADE, 2020).

Alguns aspectos relevantes associados ao período de crise sanitária, como a pandemia, foram a aceleração do desenvolvimento de recursos terapêuticos e o importante papel das modalidades de consulta, monitoramento e atendimento remotos, que foram recentemente regulamentados, passando a ser explorados como alternativa. $\mathrm{O}$ aprimoramento desses recursos aumenta as possibilidades de atuação (KARSTEN; MATTE; ANDRADE, 2020). 
Embora modalidades remotas venham sendo utilizadas com a finalidade de minimizar as perdas dos pacientes ambulatoriais (respeitando as orientações para distanciamento social durante a pandemia), há uma hesitação compreensível sobre o conhecimento das ferramentas disponíveis pelos profissionais para exercer tal tarefa de forma eficaz e que seja segura para os pacientes (GASTALDI, 2021; LEE, 2020).

Portanto, a telerreabilitação, como modalidade tão promissora quanto recente, necessita de embasamento científico e patentário das tecnologias de informação e comunicação disponíveis para que se tornem viáveis às empresas e aos profissionais do ramo, visando a possibilitar sua implantação da melhor maneira possível, maximizando seus resultados, que são os objetivos deste estudo.

\section{Metodologia}

Esta pesquisa tem caráter qualitativo exploratório-descritivo, sendo voltada para dispositivos tecnológicos que possam ser aplicados à telerreabilitação. As pesquisas exploratórias são utilizadas quando se deseja obter dados sobre a natureza de um problema, e o interesse é obter um volume de informações que explorem em profundidade como dado fenômeno ocorre. Já a pesquisa descritiva visa a identificar fatores e suas relações com a ocorrência de fenômenos com a finalidade explicar a razão das coisas (GIL, 2017).

A prospecção patentária foi feita em bases de dados nacionais e internacionais, buscando registros ligados a aplicações na telerreabilitação no período de 2011 a 2021. Para este levantamento, foi utilizado o Orbit Intelligence ${ }^{\circledR}$ (https://www.orbit.com/), que é um portal desenvolvido e gerenciado pela Questel e oferece um conjunto completo de serviços baseado na produtividade de pesquisa e na colaboração dedicada à propriedade intelectual com diversos recursos de análise, permitindo a geração e a visualização de gráficos, mapas e diagramas relativos a patentes, empresas depositantes e inventores (SEDETEC, 2014; SANTOS et al., 2021). A cobertura geográfica dessa base compreende registros de quase uma centena de países $e$ autoridades de patentes, incluindo pedidos referente à mesma invenção depositados em diferentes países, dessa forma, evitando a duplicidade dos dados gerando resultados mais específicos para o estudo prospectivo (AXONAL CONSULTORIA TECNOLÓGICA; SUZUKI, 2016; BARROS; ANJOS; BARROS, 2020; SANTOS et al., 2021). A busca patentária foi realizada nos campos título e resumo com os seguintes termos, operador booleano e caractere de truncagem: telehealth; telerehabilit+; telehealth AND physiotherap +, telerehabilitation AND physical therapy e telehealth AND physical therapy.

Além do levantamento sobre as patentes, foi feita uma prospecção bibliométrica na área para direcionar a investigação do tema e traçar um panorama das tendências. Para isso, utilizou-se as bases de dados Pubmed (https://pubmed.ncbi.nlm.nih.gov/), PEDro - Physiotherapy Evidence Database (https://pedro.org.au/) e Cochrane (https://www.cochranelibrary.com/).

Inicialmente foi feita uma busca exploratória nos campos título/resumo com os mesmos termos e período da busca patentária. Esses dados foram utilizados para se obter uma ampla visão da temática da área. Os termos selecionados para dar continuidade na pesquisa foram telerehabilitation AND physical therapy, mantendo o período (2011-2021); para afunilar a pesquisa, aplicou-se um filtro aos artigos selecionando apenas ensaios clínicos originais, para assim obter a comparação anual das patentes com as publicações científicas em telerreabilitação. 
Devido à elevada quantidade de documentos identificados, foram acrescidos critérios de seleção para proceder-se com a análise documental dos artigos científicos; portanto, delimitou-se o estudo aos manuscritos publicados nos últimos cinco anos (2016 até $1^{\circ}$ de julho 2021); que fossem ensaios clínicos originais com textos completos em indivíduos que necessitavam de assistência fisioterapêutica; que utilizaram o teleatendimento ou telemonitoramento como recurso terapêutico na reabilitação; e que possuíssem grupo controle. A busca ocorreu de 15 junho a $1^{\circ}$ de julho de 2021.

Os artigos selecionados para o estudo foram avaliados quanto à qualidade metodológica por meio dos critérios estabelecidos pela escala PEDro. Essa escala é validada e seu objetivo é classificar a qualidade dos estudos, bem como avaliar a descrição estatística, isto é, se o estudo contém informações estatísticas mínimas para que os resultados possam ser interpretáveis. A pontuação final da escala de qualidade PEDro é dada por meio da soma do número de critérios que foram classificados como satisfatórios, variando de 0 e 10 pontos (SHIWA et al., 2011). Devido à impossibilidade de realizar um ensaio clínico controlado e randomizado na área de telerreabilitação com cegamento dos terapeutas ou dos sujeitos, o escore PEDro máximo alcançável para os estudos analisados foi de 8 em 10 (SANTOS et al., 2014).

Assim, foi possível verificar quais formas de telerreabilitação estão sendo utilizadas e seus efeitos, observando-se o ano de publicação, a idade dos participantes, o país da pesquisa, a TIC utilizada e o modo de utilização (síncrona ou assíncrona).

A seleção inicial foi feita a partir da leitura dos títulos e resumos dos artigos encontrados; os artigos, cujos título e resumo não apresentavam informações suficientes, foram analisados por dois pesquisadores de maneira independente na íntegra para decidir sobre sua elegibilidade. Aqueles que apresentaram um título dentro do tema, mas o resumo não estava disponível, foram também analisados na íntegra.

Para se obter uma ampla visão da temática da área, realizou-se uma comparação entre a quantidade de patentes depositadas no período de 2011 a 2021 e a quantidade de artigos publicados em cada base de dados. Posteriormente, filtros foram aplicados para refinar estratégia de busca, conforme apresentado nos resultados (Tabela 1).

\section{Resultados e Discussão}

Como citado na metodologia, inicialmente realizou-se uma busca limitada ao período de 2011 a 2021 para se obter uma visão panorâmica de como se apresentava o cenário da temática da pesquisa. Os resultados referentes à comparação entre a quantidade de patentes depositadas no período e a quantidade de artigos publicadas em cada base de dados são apresentados na Tabela 1. 
Tabela 1 - Quantidade de depósitos de patentes e de publicações no período 2011-2021

\begin{tabular}{|c|c|c|c|c|}
\hline \multirow{2}{*}{$\begin{array}{l}\text { ESTRATÉGIA DE BUSCA } \\
\text { (TI/ABS) }\end{array}$} & \multicolumn{3}{|c|}{ Artigos } & \multirow{2}{*}{$\begin{array}{l}\text { Patentes } \\
\text { Orbit }\end{array}$} \\
\hline & Pubmed & PEDRo & Cochrane & \\
\hline Telehealth & 7161 & 60 & 2095 & 79 \\
\hline Telerehabilitat+ & 1117 & 91 & 719 & 9 \\
\hline Telehealth AND physiotherap+ & 250 & 3 & 70 & 1 \\
\hline Telerehabilitation AND physical therapy & 578 & 18 & 149 & 0 \\
\hline Telehealth AND physical therapy & 169 & 8 & 172 & 0 \\
\hline Número total de documentos & 9275 & 180 & 3205 & 89 \\
\hline
\end{tabular}

TI: Título. ABS: Abstract.

Fonte: Elaborada pelos autores deste artigo (2021)

Nota-se que há uma disparidade quando se compara o número de depósitos de patentes e de produção científica. Isso denota que as pesquisas em torno de tecnologias para a telerreabilitação têm sido substanciais. Entretanto, embora tecnologias estejam eventualmente sendo desenvolvidas, podem não estar sendo necessariamente protegidas de maneira legal.

Com relação à evolução anual das patentes e das publicações de ensaios clínicos originais, o Gráfico 1 apresenta os resultados, nele é possível perceber que houve uma evolução temporal na quantidade de produtos, sendo que 2017 foi o ano com o maior número de patentes (14) e 2020 o ano com maior número de artigos publicados (65 somando-se as 3 bases).

Gráfico 1 - Evolução anual das patentes e publicações científicas de ensaios clínicos originais em telerreabilitação 2011-2021

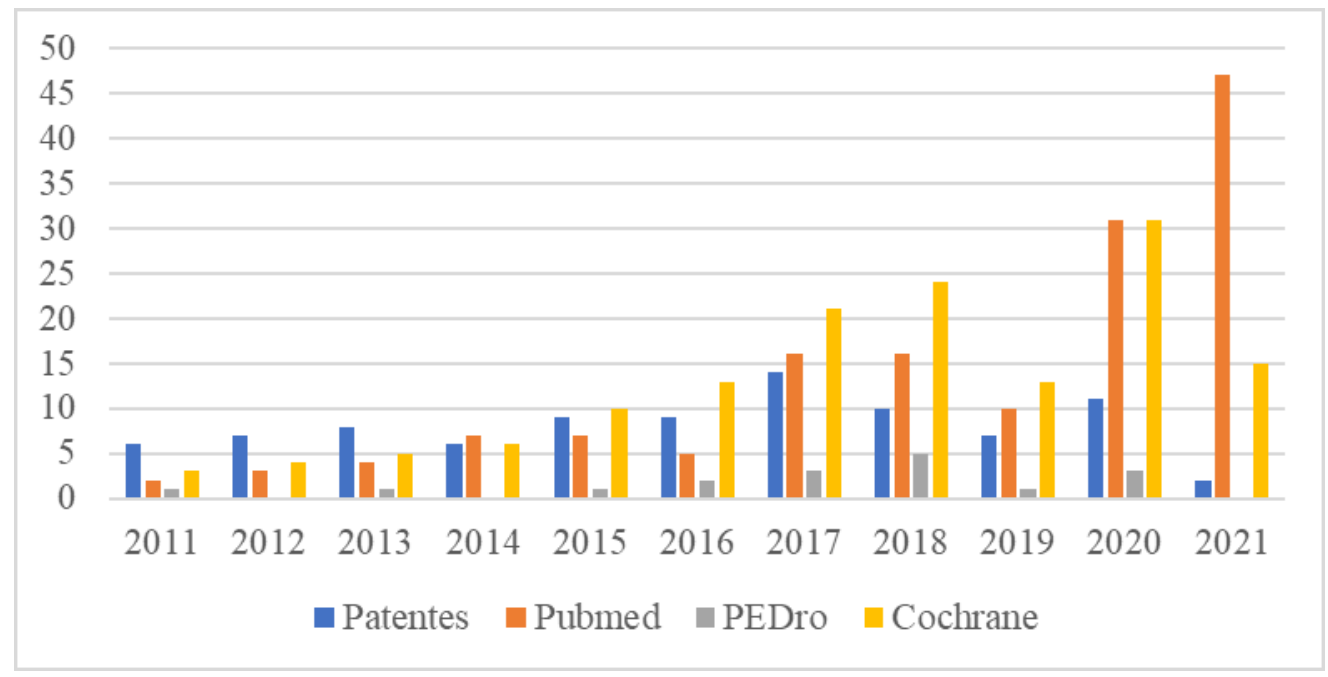

Fonte: Elaborado pelos autores deste artigo (2021)

A prospecção patentária ainda nos mostra uma série de resultados interessantes. A Tabela 2 apresenta o quantitativo de registros de patentes por país, observando-se destaque para Estados Unidos (37), Organização Europeia de Patentes (9), Índia (8), Taiwan (8) e China (6). $\mathrm{O}$ Brasil aparece com apenas um registro. Um detalhe importante a ser mencionado aqui é que uma patente pode ser registrada em mais de um país. 
Tabela 2 - Distribuição das patentes em telessaúde/telerreabilitação por país de proteção 2011-2021

$\begin{array}{cccc}\text { País } & \text { QuANTIDADE } & \text { País } & \text { QuANTIDADE } \\ \text { Alemanha } & 4 & \text { Índia } & 8 \\ \text { Austrália } & 5 & \text { Irlanda } & 4 \\ \text { Áustria } & 1 & \text { Israel } & 1 \\ \text { Brasil } & 1 & \text { Itália } & 1 \\ \text { Canadá } & 4 & \text { Japão } & 4 \\ \text { Chile } & 1 & \text { Noruega } & 1 \\ \text { China } & 6 & \text { Organização Europeia de Patentes (EPO) } & 9 \\ \text { Colômbia } & 1 & \text { Organização Mundial da } & 4 \\ \text { Coreia do Sul } & 5 & \text { Propriedade Intelectual (WIPO) } & 1 \\ \text { Dinamarca } & 1 & \text { Peru } & 1 \\ \text { Equador } & 1 & \text { Polônia } & 4 \\ \text { Espanha } & 1 & \text { Reino Unido } & 1 \\ \text { Estados Unidos } & 37 & \text { Rússia } & 1 \\ \text { Filipinas } & 1 & \text { Suécia } & 4 \\ \text { Finlândia } & 1 & \text { Suíça } & 8 \\ \text { França } & 1 & \text { Taiwan } & 1 \\ \text { Hong Kong } & 1 & \text { Turquia } & \end{array}$

Nota: patentes podem ser protegidas em um ou mais países.

Fonte: Elaborada pelos autores deste artigo, dados Orbit (2021)

Com relação ao cenário dos países, o Índice Global de Inovação (2020) é o resultado de uma colaboração entre a Universidade Cornell, o Instituto Europeu de Administração de Empresas (INSEAD) e a Organização Mundial da Propriedade Intelectual (OMPI) e mostra que a Suíça é o país mais inovador do mundo, seguido pela Suécia, Estados Unidos da América, Reino Unido e Holanda (CORNELL UNIVERSITY; INSEAD; WIPO, 2020).

Em referência à América do Sul, esta mesma publicação explica que, com baixos insumos de inovação, a região também luta para traduzi-los com eficácia em resultados. Apenas Chile, Uruguai e Brasil produzem altos níveis de artigos científicos e técnicos, e apenas o Brasil ocupa uma posição elevada em matéria de patentes por origem; mesmo assim, aparece na $62^{a}$ posição do ranking mundial, ficando atrás de países como Chipre e Mongólia. Ferreira, Guimarães e Contador (2009) reforçam essa deficiência mostrando que, frente a um mercado globalizado, competitivo e repleto de inovações tecnológicas, as empresas brasileiras ainda não atentaram para a importância da utilização de patentes como instrumento competitivo, assim como não atentaram para a importância da exploração das patentes como fonte de informação tecnológica.

A única proteção no Brasil tem o título "Monitoramento de gravidez e nascimento de atendimento de telessaúde de múltiplos fatores" e é de um sistema de monitoramento de gestação de alto risco, em formato de adesivo, usado pela gestante. Essa proteção possui um sensor de som que detecta o som vascular das artérias umbilicais do feto ou das artérias uterinas da gestante. O sensor é conectado a uma unidade de processamento, que executa um algoritmo e extrai um parâmetro de sinal, que pode ser transmitido por sinal de áudio, por display visual ou então enviar uma mensagem. Essa patente teve seu depósito em 2012 e publicação nacional em 2017 (DINESEN; RIKNAGEL; STRUIJK, 2012). 
Esse déficit de solicitações de patentes pode indicar a ausência ou baixa capacidade da indústria brasileira para reproduzir as tecnologias, evidenciando o estado de dependência tecnológica nacional nessa área (SILVA; MENDONÇA, 2019).

A WIPO e o Instituto Nacional da Propriedade Industrial (INPI) adotam uma classificação de patentes de acordo com a área tecnológica a que pertencem, que é a Classificação Internacional de Patentes (CIP). Esta tem como objetivo inicial o estabelecimento de uma ferramenta de busca eficaz para a recuperação de documentos de patentes pelos escritórios de propriedade intelectual e demais usuários, a fim de estabelecer a novidade e avaliar a atividade inventiva de divulgações técnicas em pedidos de patente (OMPI, 1999). Com relação a essa classificação, as patentes localizadas neste estudo pertencem às áreas constantes da Tabela 3.

Tabela 3 - Classificação Internacional das Patentes (CIP) em telessaúde/telerreabilitação (2011-2021)

\begin{tabular}{cccc} 
Código & SEÇão & ClASSE & QUANTIDADE \\
A61 & Necessidades humanas & Ciência médica ou veterinária; higiene & 806 \\
A63 & Necessidades humanas & Esportes; jogos; diversões & 5 \\
G01 & Física & Medição; teste & 6 \\
G06 & Física & Cômputo; cálculo ou contagem & 751 \\
G16 & Física & Tecnologia de informação e comunicação especial & 796 \\
H04 & Eletricidade & adaptada para campos de aplicação específicos & 325 \\
\hline
\end{tabular}

Nota: as patentes são classificadas em uma ou mais seções, classes ou subclasses da CIP.

Fonte: Elaborada pelos autores deste artigo, dados Orbit (2021)

Analisando esses resultados, percebe-se que há uma concentração nas áreas de ciência médica, cômputo e tecnologias de informação e comunicação. Essa concentração de patentes conecta-se com o que foi encontrado nos artigos científicos, em que grande parte das publicações apresenta o uso de tecnologias de informação e comunicação aplicadas à telerreabilitação, com a utilização de dispositivos habilitados para internet (como desktops, laptops, tablets e smartphones) que permitam acesso a softwares, aplicativos e sites que irão proporcionar o contato entre o paciente e o profissional de maneira síncrona ou o contato entre o paciente $e$ alguma interface amigável que foi programada previamente pelo profissional e fica disponível para acesso a qualquer momento (interação assíncrona).

A prospecção patentária ainda nos mostra que há certa concentração em algumas empresas, sendo que, na temática pesquisada, as cinco empresas que mais detêm registros são a Philips com cinco, a Robert Bosch com quatro, a Teladoc Health com quatro e a Jonata Sub Two e Robert Bosch Healthcare Systems, ambas com três depósitos.

Desde 2016, a Philips conduz pesquisas para abordar desafios globais de saúde e construção eficiente e eficaz de sistemas de saúde, sendo que o foco são as ferramentas digitais e o atendimento conectado à tecnologia, que podem desempenhar um papel mais acessível, cuidados de saúde integrados e sustentáveis (FUTURE HEALTH INDEX, 2021).

Empresas brasileiras não aparecem na listagem. Pires e colaboradores (2021) realizaram o mapeamento de patentes de respiradores artificiais ou ventiladores mecânicos e identificaram que, de 6.394 famílias de patentes, somente 14 famílias de patentes de residentes brasileiros, evidenciando a necessidade de ampliar os estudos que garantam a evolução científico-tecnológica desses dispositivos no país, o que também reforça os achados de Ferreira, Guimarães e Contador (2009). 
O domínio da tecnologia que mais apareceu foi a tecnologia de computador (36), métodos de TI para gestão (35) e tecnologia médica (33). A comunicação digital, telecomunicação e tecnologia audiovisual apresentaram a ocorrência de 12, 10 e três, respectivamente.

Sobre a situação dos pedidos de patentes, $38,2 \%$ são concedidas, $33,7 \%$ são pendentes, $25,8 \%$ expiradas e $2,3 \%$ revogadas.

No tocante à pesquisa bibliométrica, a Tabela 4 apresenta uma sumarização dos estudos selecionados após os critérios de elegibilidade. Esses artigos foram classificados quanto à qualidade dos estudos pela escala de qualidade PEDro, e a maioria dos estudos selecionados neste estudo apresentou alta qualidade metodológica, ou seja, escore $\geq 6$ (75\% do escore máximo possível) (SANTOS et al., 2014).

Tabela 4 - Caracterização dos estudos científicos em telerreabilitação (2016-2021)

\begin{tabular}{ccccccc}
$\begin{array}{c}\text { Autor } \\
\text { PRINCIPAL }\end{array}$ & ANo & PAís & $\begin{array}{c}\text { TEMPO DE } \\
\text { INTERVENção }\end{array}$ & $\begin{array}{c}\text { AMOSTRA } \\
\text { IDADE }\end{array}$ & $\begin{array}{c}\text { EsCALA } \\
\text { PEDRO }\end{array}$ \\
Azma & 2017 & Irã & 6 semanas & 54 & $58,25 \pm 7,41$ & 6 \\
Bao & 2018 & Estados Unidos & 8 semanas & 12 & $75,6 \pm 4,9$ & 8 \\
Bell & 2020 & Estados Unidos & 10 semanas & 38 & $64,4 \pm 8,2$ & 7 \\
Bennell & 2017 & Austrália & 24 semanas & 168 & $61.1 \pm 6.9$ & 8 \\
Bettger & 2020 & Estados Unidos & 12 semanas & 306 & $65.4 \pm 7.7$ & 6 \\
Bini & 2016 & Estados Unidos & até a alta & 29 & 63,6 & 6 \\
Blanquero & 2020 & Espanha & 4 semanas & 74 & $45 \pm 11$ & 6 \\
Chae & 2020 & Coreia do Sul & 12 semanas & 23 & $64.5 \pm 9.6$ & 3 \\
Chen & 2017 & China & 12 semanas & 54 & $66.52 \pm 12.08$ & 8 \\
Chen & 2020 & Taiwan & 12 semanas & 15 & $53,0 \pm 6,2$ & 7 \\
Egmond & 2020 & Holanda & 12 semanas & 52 & $64,5 \pm 6,7$ & 4 \\
Fjeldstad-pardo & 2018 & Estados Unidos & 8 semanas & 30 & $54,7 \pm 12,3$ & 7 \\
Galiano-Castillo & 2016 & Espanha & 8 semanas & 81 & $47.4 \pm 9.6$ & 8 \\
Gondim & 2017 & Brasil & 12 semanas & 28 & $65 \pm 8$ & 7 \\
Hwang & 2017 & Austrália & 12 semanas & 53 & $67 \pm 12$ & 8 \\
Kalron & 2018 & Israel & 6 semanas & 32 & $65.7 \pm 7.8$ & 7 \\
Kloek & 2018 & Holanda & 12 semanas & 208 & $63.8 \pm 8.5$ & 6 \\
Nelson & 2020 & Austrália & 6 semanas & 70 & 67 & 7 \\
Pastora-Bernal & 2018 & Espanha & 12 semanas & 18 & $52.5 \pm 10.5$ & 8 \\
Paul & 2018 & Reino unido & 24 semanas & 90 & $56.1 \pm 9.6$ & 6 \\
Rothgangel & 2018 & Alemanha & 6 semanas & 75 & $61.1 \pm 14.2$ & 8 \\
\hline
\end{tabular}

Fonte: Elaborada pelos autores deste artigo (2021)

Pode-se constatar um predomínio de estudos norte-americanos (5), seguidos de australianos (3), espanhóis (3) e holandeses (2). Além disso, os anos mais frequentes de publicação são 2018 (7), 2020 (6) e 2017 (5), o que deixa evidente o interesse recente na realização de pesquisas 
relacionadas à telerreabilitação. Os estudos somam 1.510 pacientes, com intervenções que variaram de quatro a 24 semanas.

A idade média dos participantes foi de 61,6 anos, o que, de certa forma, é surpreendente, pois mostra que o público de idade mais avançada conseguiu participar e concluir diversas pesquisas, nos mais diversos países e continentes com sucesso. Takemoto et al. (2018) relatam que, embora os adultos mais velhos sejam frequentemente percebidos como sem interesse $e$ capacidade de adotar tecnologias, estudos recentes mostram que eles se sentem confortáveis em adotar a tecnologia e que a aceitação do usuário é alta com treinamento adequado e facilitação orientada. Holthe et al. (2018) complementam essa informação ressaltando que até mesmo a população mais idosa com algum grau de comprometimento cognitivo ou demência pode ter familiaridade e se beneficiar com o uso de tecnologias, o que mostra um potencial gigantesco para o uso da telerreabilitação. Observou-se uma grande heterogeneidade das pesquisas selecionadas em vários aspectos: tema abordado, faixa etária, tamanho da amostra, tipo de tecnologia utilizada, tempo de intervenção e seguimento.

Sobre as tecnologias de informação e comunicação, as publicações mostraram diversas formas de utilização, como pode ser verificado na Tabela 5.

Tabela 5 - Caracterização das TICs utilizadas nos estudos científicos em telerreabilitação (2016-2021)

\begin{tabular}{|c|c|c|c|c|}
\hline \multirow{2}{*}{ Autor } & \multirow{2}{*}{ Ano } & \multicolumn{3}{|c|}{ TICs } \\
\hline & & EQUIPAMENTO & Programa/APLicativo/Site & COMUNICAÇÃO \\
\hline Azma & 2017 & smartphone & - & síncrona \\
\hline Bao & 2018 & ipod, motor & software SDK & assíncrona \\
\hline Bell & 2020 & $\begin{array}{l}\text { smartphone, acelerômetro } \\
\text { tri-axial, giroscópio, sensores, } \\
\text { magnetômetro, câmeras }\end{array}$ & $\begin{array}{l}\text { aplicativo móvel, plataforma } \\
\text { eletrônica com } \\
\text { exercícios, software motive tracker }\end{array}$ & assíncrona \\
\hline Bennell & 2017 & smartphone & - & síncrona \\
\hline Bettger & 2020 & $\begin{array}{l}\text { dispositivo VERA } \\
\text { (tela + câmera) }\end{array}$ & $\begin{array}{l}\text { fisioterapeuta/treinador } \\
\text { avatar, software VERA } \\
\text { (assistente de reabilitação } \\
\text { de exercícios virtuais) }\end{array}$ & ambas \\
\hline Bini & 2016 & iPod touch & software CaptureProof & assíncrona \\
\hline Blanquero & 2020 & tablet & software ReHand & assíncrona \\
\hline Chae & 2020 & smartwatch, smartphone & $\begin{array}{c}\text { aplicativos desenvolvidos para o estudo, } \\
\text { rede neural convolucional e algoritmo } \\
\text { de aprendizado de máquina }\end{array}$ & síncrona \\
\hline Chen & 2017 & $\begin{array}{l}\text { aparato para telerreabilitação } \\
\text { (audio+vídeo) } \\
\text { eletromiógrafo e dispositivo } \\
\text { que capta sinais vitais }\end{array}$ & - & síncrona \\
\hline Chen & 2017 & $\begin{array}{l}\text { biofeedback eletromiográfico, } \\
\text { dispositivos de coleta de } \\
\text { dados fisiológicos, } \\
\text { processador de } \\
\text { sinais biológicos }\end{array}$ & sistema de dados de rede & assíncrona \\
\hline
\end{tabular}




\begin{tabular}{|c|c|c|c|c|}
\hline \multirow{2}{*}{ Autor } & \multirow{2}{*}{ AnO } & \multicolumn{3}{|c|}{ TICs } \\
\hline & & EQUIPAMENTO & Programa/APLicativo/Site & Comunicação \\
\hline Chen & 2020 & $\begin{array}{c}\text { smartphone, tablet, } \\
\text { dispositivo de sensor } \\
\text { de movimento } \\
\text { (vestível), sensores baseados } \\
\text { em IMU vestíveis, }\end{array}$ & $\begin{array}{l}\text { aplicativos móveis Patient } \\
\text { app e Doctor app }\end{array}$ & assíncrona \\
\hline Egmond & 2020 & $\begin{array}{l}\text { dispositivo habilitado } \\
\text { para internet }\end{array}$ & plataforma eletrônica com exercícios & ambas \\
\hline $\begin{array}{l}\text { Fjeldstad- } \\
\text { pardo }\end{array}$ & 2018 & $\begin{array}{l}\text { dispositivo habilitado } \\
\text { para internet }\end{array}$ & plataforma de viodeoconferência & síncrono \\
\hline $\begin{array}{l}\text { Galiano- } \\
\text { Castillo }\end{array}$ & 2016 & $\begin{array}{l}\text { dispositivo habilitado } \\
\text { para internet }\end{array}$ & $\begin{array}{c}\text { programa de telerreabilitação } \\
\text { e-CUIDATE system, } \\
\text { personalizado para cada paciente }\end{array}$ & ambas \\
\hline Gondim & 2017 & smartphone & $\begin{array}{l}\text { manual de exercícios do } \\
\text { programa pró-Parkinson }\end{array}$ & ambas \\
\hline Hwang & 2017 & $\begin{array}{l}\text { dispositivo habilitado } \\
\text { para internet }\end{array}$ & $\begin{array}{l}\text { plataforma de viodeoconferência, } \\
\text { compartilhamento de telas, } \\
\text { chat, slides eletrônicos com } \\
\text { arquivos de áudio embutidos }\end{array}$ & ambas \\
\hline Kalron & 2018 & $\begin{array}{l}\text { dispositivo habilitado } \\
\text { para internet }\end{array}$ & plataforma com videoclipes de exercícios & ambas \\
\hline Kloek & 2018 & $\begin{array}{l}\text { dispositivo habilitado } \\
\text { para internet }\end{array}$ & plataforma eletrônica com exercícios & assíncrona \\
\hline Nelson & 2020 & tablet & $\begin{array}{l}\text { plataforma eletrônica e programa } \\
\text { de exercícios em tablet }\end{array}$ & ambas \\
\hline $\begin{array}{l}\text { Pastora- } \\
\text { Bernal }\end{array}$ & 2018 & $\begin{array}{l}\text { dispositivo habilitado } \\
\text { para internet }\end{array}$ & $\begin{array}{c}\text { aplicativo com acesso a vídeos, imagens } \\
\text { e parâmetros dos exercícios }\end{array}$ & ambas \\
\hline Paul & 2018 & $\begin{array}{l}\text { dispositivo habilitado } \\
\text { para internet }\end{array}$ & plataforma eletrônica com exercícios & assíncrona \\
\hline Rothgangel & 2018 & tablet & $\begin{array}{l}\text { plataforma eletrônica com } \\
\text { exercícios, realidade aumentada }\end{array}$ & assíncrona \\
\hline
\end{tabular}

Fonte: Elaborada pelos autores deste artigo (2021)

O modelo assíncrono foi o mais utilizado pelos autores. Segundo Tousignant et al. (2011), esse modelo se destaca porque cria um registro digital acessível que pode ser facilmente acessado. Assim, cria-se uma experiência que potencializa os pontos fortes do meio digital.

Fica claro ao observar a Tabela 5 o universo de possibilidades que a telerreabilitação oferece, em que diversos dispositivos ou formas distintas do uso das TICs podem ser usados a favor dos pacientes, usando a inovação como forma de trazer conforto e qualidade de vida à população que necessita de assistência fisioterapêutica. Nesse contexto, as principais abordagens dos autores encontrados na prospecção bibliométrica é relatada a seguir.

Bettger et al. (2020) examinaram o efeito de um programa de fisioterapia virtual nos custos de saúde e nos resultados clínicos em comparação com o atendimento tradicional após artroplastia total de joelho em 306 pacientes, tendo descoberto que a telerreabilitação reduziu significativamente os custos (média de \$2.745 a menos), com a mesma eficácia das outras intervenções, apenas com o detalhe de haver um maior relato de quedas nesse grupo. Um sistema 
(Virtual Exercise Rehabilitation Assistant - VERA), composto de tela e câmera (portáteis), que funciona com o uso de tecnologia de rastreamento, foi disponibilizado para ficar na casa de cada paciente, em que o fisioterapeuta/treinador virtual demonstra e orienta as atividades que o paciente deve fazer, com feedback imediato sobre a qualidade do exercício.

O complexo estudo de Chae et al. (2020) desenvolveu e avaliou a eficácia de um sistema de reabilitação domiciliar em sobreviventes de acidente vascular encefálico (AVC), que pode reconhecer e registrar o tipo e a frequência dos exercícios de reabilitação realizados pelo usuário, usando um relógio (smartwatch) e um aplicativo para smartphone equipado com um algoritmo de aprendizado de máquina. Esse estudo descobriu que um sistema de atendimento domiciliar usando um smartwatch comercial e modelo de aprendizado de máquina pode facilitar a participação no treinamento doméstico e melhorar escores funcionais e físicos no tratamento de pacientes com AVC crônico. Essa estratégia pode ser uma ferramenta econômica para o tratamento domiciliar de sobreviventes de AVC no futuro.

No estudo espanhol de Pastora-Bernal et al. (2018), o objetivo foi avaliar a viabilidade e a eficácia de uma intervenção de telerreabilitação personalizável após a descompressão subacromial artroscópica e compará-la com os cuidados tradicionais. O paciente tinha acesso a aplicativo com vídeos, imagens e parâmetros dos exercícios (primeiro sozinho e acompanhado com fisioterapeuta depois). Os autores sugerem que há evidências da eficácia da telerreabilitação pós-operatória na síndrome do impacto do ombro, não sendo inferior à fisioterapia tradicional.

Nelson et al. (2020), por sua vez, estudaram se o atendimento via telerreabilitação é tão eficaz quanto os cuidados de fisioterapia em pessoas após a artroplastia total do quadril em 70 pessoas. $\mathrm{O}$ grupo de controle recebia internação convencional de fisioterapia e um programa de exercícios para casa (cartilha impressa). O grupo de telerreabilitação recebeu atendimento remotamente em suas casas e um programa de exercícios domésticos baseado em tecnologia (usando aplicativos para iPad). Como resultado, contatou-se que a telerreabilitação pode ser realizada para pacientes com artroplastia total de quadril, em suas próprias casas, usando tecnologia prontamente disponível, mantendo altos níveis de satisfação.

Kloek et al. (2018) investigaram o custo-efetividade de uma intervenção fisioterapêutica mista (mescla de atendimentos presenciais com exercícios via plataforma eletrônica - https:// www.e-exercise.nl denominada e-Exercício) em comparação com a fisioterapia usual em pacientes com osteoartrite de quadril e/ou joelho, tanto do ponto de vista social quanto de saúde. $\mathrm{O}$ e-Exercício foi significativamente mais barato, mas não teve relação custo-efetivo do ponto de vista social e também de saúde. Assim, eles concluíram que a decisão sobre qual intervenção escolher pode ser baseada nas preferências do paciente e do fisioterapeuta.

A pesquisa de Kalron et al. (2018) avaliou os efeitos da telerreabilitação na mobilidade em pessoas após cirurgia de quadril, comparando a telerreabilitação baseada em videoclipes de exercícios (com foco nos membros inferiores) com grupo controle (livreto de exercícios). Durante o acompanhamento, o grupo de telerreabilitação continuou a melhorar em todas as medidas de resultado, em contraste com o grupo de controle, que não mostrou alterações em cinco das seis medidas de resultado. Assim, sugeriram que a telerreabilitação, um tratamento complementar à fisioterapia padrão, gera um efeito positivo na mobilidade das pessoas após a cirurgia de quadril.

Um estudo brasileiro de Gondim et al. (2017) buscou avaliar os efeitos da orientação individualizada e do monitoramento por telefone em um programa de exercícios terapêuticos 
domiciliares autossupervisionados (cartilha) sobre os sinais e sintomas da Doença de Parkinson. Como resultado, eles relataram que a orientação individualizada e o monitoramento semanal por telefone em um programa de exercícios terapêuticos domiciliares autossupervisionados apresentaram efeitos positivos em estágios iniciais da DP quando comparados a um grupo de cuidados usuais.

Já a pesquisa de Galiano-Castillo et al. (2016) objetivou investigar a eficácia de um sistema de telessaúde em sobreviventes de câncer de mama, por meio do Cuidate, um sistema baseado na internet com exercícios personalizados três vezes/semana, respeitando os padrões do Colégio Americano de Medicina do Esporte para sobreviventes de câncer. Segundo os autores, de acordo com os resultados encontrados em grupo de intervenção com 40 pessoas, esse programa pode melhorar os efeitos adversos e manter os benefícios em sobreviventes do câncer de mama. Ele afirmam ainda que os resultados desse estudo têm implicações encorajadoras para o tratamento do câncer. Um detalhe que chama a atenção é que a satisfação global com a intervenção remota foi de $97,8 \%$.

Chen et al. (2020) pesquisaram sobre os efeitos da telerreabilitação sobre a função física de sobreviventes de AVC com hemiplegia, com atendimentos síncronos realizando estimulação neuromuscular desencadeada por eletromiografia (ETNS) por eletromiógrafo portátil disponibilizado aos pacientes. Concluíram que a telerreabilitação é provavelmente tão eficaz quanto a reabilitação ambulatorial convencional para melhorar recuperação funcional em sobreviventes de AVC, podendo inclusive aliviar a carga dos cuidadores.

Rothgangel et al. (2018) conduziram um estudo multicêntrico em amputados sobre os efeitos da terapia de espelho tradicional (25 pacientes) com um teletratamento centrado no paciente (plataforma PACT, 26 pacientes), além de exercícios sensoriomotores sem espelho na dor do membro fantasma (24 pacientes). O teletratamento foi aplicado por seis semanas após quatro semanas de terapia de espelho convencional e não apresentou efeitos adicionais. A plataforma PACT (ou telerreabilitação centrada no paciente) foi desenvolvida pelos mesmos autores em um estudo prévio.

De forma geral, a maioria dos estudos incluídos demonstrou que a telerreabilitação é capaz de produzir resultados superiores ou semelhantes quando o tratamento é comparado aos métodos de reabilitação tradicionais. Também foram observados altos índices de satisfação, maior flexibilidade, menos deslocamentos necessários e tempos de espera reduzidos.

\section{Considerações Finais}

Este artigo apresentou um estudo de prospecção científica e tecnológica de TICs relacionadas à telerreabilitação por meio de levantamento científico e patentário.

Observou-se que a pandemia acelerou mudanças radicais necessárias na prestação de cuidados aos pacientes e também em provedores (com investimentos em telessaúde e atendimento virtual).

Os resultados encontrados demonstram que o uso das TICs na telerreabilitação mostra-se bastante promissor, considerando-se o crescente interesse na temática, sendo que as prospecções realizadas mostraram uma série de dispositivos, ferramentas, aplicativos, softwares, entre outros, que, em seu conjunto, trazem diversas possibilidades de atuação para os profissionais, 
que, por sua vez, serão traduzidas em uma assistência mais eficiente aos pacientes. Além disso, verificou-se que a quantidade de pedidos de patente depositados no Brasil é muito inferior quando em comparação com outros países, um contraposto com os EUA que possui a maior quantidade de registros nessa área. É notória a dependência brasileira no mercado de patentes de inovação tecnológica em função de empresas e/ou instituições de pesquisa localizadas no exterior.

Contudo, o trabalho mostrou que o desenvolvimento de estudos e de tecnologias de soluções para a problemática do atendimento remoto é diversificada, que há opções de dispositivos para os profissionais oferecerem saúde de qualidade e que ainda existe um campo vasto para o desenvolvimento de inovações.

\section{Perspectivas Futuras}

Esse é um tema que desperta muito interesse e curiosidade na comunidade de profissionais de saúde por ser relativamente novo e ter sido catapultado em importância em virtude da pandemia de Covid-19. Nessa perspectiva, é importante pensar que a implantação da telerreabilitação como forma de intervenção usual ainda demorará um pouco para se estabelecer, pois requer capacitação profissional adequada para a modalidade e acesso satisfatório à internet por parte dos profissionais e pacientes, realidade restrita apenas para parte da população brasileira, sendo mais acessível em países desenvolvidos.

Com o aumento da cobertura das TICs, o custo pode ser diluído pelo número de usuários, o que possibilita e facilita o desenvolvimento de tais inovações tecnológicas que propiciam a telerreabilitação.

Ainda é preciso pensar na facilidade da interação relacionada à interface homem/máquina, em que os softwares e aplicativos são cada vez mais intuitivos e fáceis de manusear. Há os casos em que há avatares (profissionais virtuais) que buscam simular ao máximo as ações do "profissional real", com o intuito de obter os mesmos efeitos de uma intervenção convencional. Sem dúvida, o passar do tempo irá aperfeiçoar essa realidade virtual; se considerarmos que a habilidade humana cresceu ao longo da história, a Lei de Moore nos mostra que a tecnologia evolui muito rápido (dobrando as capacidades a cada 18 ou 24 meses, dependendo do segmento).

Para finalizar, uma pergunta nos inquieta: Será que chegaremos ao dia em que a tecnologia ficará mais eficiente que a intervenção humana? Ou seja, em algum momento, o profissional não será mais necessário? Sem dúvida, o tempo nos mostrará as respostas para esses questionamentos.

\section{Referências}

AXONAL CONSULTORIA TECNOLÓGICA; SUZUKI, H. Orbit.com: Visão Geral Sobre o Sistema. 2016. Disponível em: Axonal.https://axonal.com.br/arquivos/PDF/Orbit VisaoGeralSistemaPARTES1a3BUSCAVISUALIZACAOSELECAO.pdf. Acesso em: 10 ago. 2021.

AZMA, K. et al. Efficacy of telerehabilitation compared with office-based physical therapy in patients with knee osteoarthritis: A randomized clinical trial. J Telemed Telecare, [s.l.], v. 0, n. 0, p. 1-6, 2017. DOI: https://doi.org/10.1177/1357633X17723368. 
$\mathrm{BAO}$, T. et al. Effects of long-term balance training with vibrotactile sensory augmentation among community-dwelling healthy older adults: a randomized preliminary study. J. Neuroeng. Rehabilitation, [s.l.], v.15, n.5, 13p., 2018. DOI: https://doi.org/10.1186/s12984-017-0339-6.

BARROS, G. M.; ANJOS, M. S.; BARROS, G. M. Prospecção tecnológica do pequizeiro. Res. Soc. Dev. [s.l.], v. 9, n. 9, p 1-15, 2020. DOI: http://dx.doi.org/10.33448/rsd-v9i9.7957.

BELL, K. M. A Portable System for Remote Rehabilitation Following a Total Knee Replacement: A Pilot Randomized Controlled Clinical Study. Sensors, [s.l.], v. 20, n. 21, p. 6.118, 2020. DOI: https:// doi.org/10.3390/s20216118.

BENNELL, K. L. et al. Telephone Coaching to Enhance a Home-Based Physical Activity Program for Knee Osteoarthritis: A Randomized Clinical Trial. Arthritis Care Res, [s.l.], v. 69, n. 1, p. 84-89, 2016. DOI: https://doi.org/10.1002/acr.22915.

BETTGER, J. et al. Effects of Virtual Exercise Rehabilitation In-Home Therapy Compared with Traditional Care After Total Knee Arthroplasty VERITAS, a Randomized Controlled Trial. J Bone Joint Surg Am., [s.l.], v. 102, n. 2, p. 101-109, 2020. DOI: https://doi.org/10.2106/JBJS.19.00695.

BINI, S.A.; MAHAJAN, J. Clinical outcomes of remote asynchronous telerehabilitation are equivalent to traditional therapy following total knee arthroplasty: A randomized control study. J Telemed Telecare, [s.l.], v. 0, n. 0, p. 1-9, 2016. DOI: https://doi.org/10.1177/1357633X16634518.

BLANQUERO, J. et al. Feedback-guided exercises performed on a tablet touchscreen improve return to work, function, strength and healthcare usage more than an exercise program prescribed on paper for people with wrist, hand or finger injuries: a randomised trial. J Physiother, [s.l.], v. 66, n. 4, p. 236-242, 2020. DOI: https://doi.org/10.1016/j.jphys.2020.09.012.

BONNEVIE, T. et al. Advanced telehealth technology improves home-based exercise therapy for people with stable chronic obstructive pulmonary disease: a systematic review. J Physiother, [s.l.], v. 67, n. 1, p. 27-40, 2021. DOI: https://doi.org/10.1016/j.jphys.2020.12.006.

\section{BRASIL. Diretrizes Metodológicas avaliação de desempenho de tecnologias em saúde} Desinvestimento e Reinvestimento. Brasília, DF: Ministério da saúde, 2016. 47p. Disponível em: http://conitec.gov.br/images/ArtigosPublicacoes/Diretrizes/DIRETRIZ_AdTS_final_ISBN.pdf. Acesso em: 20 ago. 2021.

CHAE, S. H. et al. Development and Clinical Evaluation of a Web-Based Upper Limb Home Rehabilitation System Using a Smartwatch and Machine Learning Model for Chronic Stroke Survivors: Prospective Comparative Study. JMIR Mhealth Uhealth, [s.l.], v. 8, n. 7, 2020. DOI: https://doi.org/10.2196/17216.

CHEN, J. Effects of Home-based Telesupervising Rehabilitation on Physical Function for Stroke Survivors with Hemiplegia. Am J Phys Med Rehabil, [s.l.], v. 96, n. 3, 2017. DOI: https://doi. org/10.1097/PHM.0000000000000559.

CHEN, Y. et al. Wearable Motion Sensor Device to Facilitate Rehabilitation in Patients With Shoulder Adhesive Capsulitis: Pilot Study to Assess Feasibility. J. Medical Internet Res., [s.l.], v. 22, n. 7, 2020. DOI: https://doi.org/10.2196/17032.

CORNELL UNIVERSITY; INSEAD; WIPO. The Global Innovation Index 2020: Who Will Finance Innovation? Ithaca, Fontainebleau, and Geneva. 2020. Disponível em: https:/www.wipo.int/edocs/ pubdocs/en/wipo_pub_gii_2020.pdf. Acesso em: 20 ago. 2021. 
DINESEN, B. R.; RIKNAGEL, D. K. T.; STRUIJK, J. J. S. Monitoramento de gravidez e nascimento de atendimento de telessaúde de múltiplos fatores. Depositante: Aalborg University. Procurador: André Luiz Alvarez. DK2012/050439. Depósito: 30 nov. 2012. Concessão: 13 jun. 2017.

FERREIRA, A. A.; GUIMARÃES, E. R.; CONTADOR, J. C. Patente como instrumento competitivo e como fonte de informação tecnológica. Gest. Prod., [s.l.], v. 16, n. 2, p. 209-221, 2009. DOI: https://doi.org/10.1590/S0104-530X2009000200005.

FIORATTI, I. et al. A pandemia de COVID-19 e a regulamentação do atendimento remoto no Brasil: novas oportunidades às pessoas com dor crônica. BrJP. São Paulo, v. 3, n. 2, p. 193-194, 2020. DOI: 10.5935/2595-0118.20200039.

FJELDSTAD-PARDO, C.; THIESSEN, A.; PARDO, G. Telerehabilitation in Multiple Sclerosis: Results of a Randomized Feasibility and Efficacy Pilot Study. Int J Telerehabil., [s.l.], v. 10, n. 2, p. 55-64, 2018. DOI: https://doi.org/10.5195/ijt.2018.6256.

FUTURE HEALTH INDEX. A resilient future Healthcare leaders look beyond the crisis. 2021. Disponível em: https:/www.philips.com/c-dam/corporate/newscenter/global/future-health-index/ report-pages/experience-transformation/2021/philips-future-health-index-2021-report-healthcareleaders-look-beyond-the-crisis-global.pdf. Acesso em: 20 ago. 2021.

GALIANO-CASTILLO, N. et al. Telehealth system: A randomized controlled trial evaluating the impact of an internet-based exercise intervention on quality of life, pain, muscle strength, and fatigue in breast cancer survivors. Cancer, [s.l.], v. 122, n. 20, p. 3.166-3.174, 2016. DOI: https://doi.org/ $10.1002 /$ cncr.30172.

GASTALDI, A. C. Fisioterapia e os desafios da Covid-19. Fisioter. Pesqui., São Paulo, v. 28, n. 1, 2021. DOI: https://doi.org/10.1590/1809-2950/00000028012021.

GIL, A. C. Como Elaborar Projetos de Pesquisa. 6. ed. São Paulo: Atlas, 2017.

GONDIM, I. T. G. O. et al. Individualized guidance and telephone monitoring in a selfsupervised home-based physiotherapeutic program in Parkinson. Fisioter. Mov., Curitiba, v. 30, n. 3, p. 559568, 2017. DOI: http://dx.doi.org/10.1590/1980-5918.030.003.AO14.

GONZALEZ-GEREZ, J. J. et al. Therapeutic pulmonar telerehabilitation protocol for patients affected by COVID-19, confined to their homes: study protocol for a randomized controlled trial. Trials. [s.l.], v. 21, n. 588, 9p., 2020. DOI: https://doi.org/10.1186/s13063-020-04494-w.

GREENHALGH, T.; KOH, G. C. H.; CAR, J. Covid-19: a remote assessment in primary care. BMJ. [s.l.], v. 368, 2020. DOI: https://doi.org/10.1136/bmj.m1182.

HOLTHE, T, et al. A. Usability and acceptability of technology for community-dwelling older adults with mild cognitive impairment and dementia: a systematic literature review. Clin Interv Aging, [s.l.], v. 4, n. 13, p. 863-886, 2018.

HWANG, R. Home-based telerehabilitation is not inferior to a centre-based program in patients with chronic heart failure: a randomised trial. J Physiother, [s.l.], v. 63, n. 2, p. 101-107, 2017. DOI: https://doi.org/10.1016/j.jphys.2017.02.017.

KALRON, A. et al. Effect of telerehabilitation on mobility in people after hip surgery: a pilot feasibility study. Int J Rehabil Res., [s.l.], v. 41, n. 3, p. 244-250, 2018. DOI: https://doi.org/10.1097/

MRR.0000000000000296. PMID: 29794545. 
KARSTEN, M.; MATTE, D. L.; ANDRADE, F. M. D. A pandemia da COVID-19 trouxe desafios e novas possibilidades para a Fisioterapia no Brasil: estamos preparados? Rev. Pesqui. Fisioter., São Paulo, v. 10, n. 2. p. 142-145. 2020. DOI: https://doi.org/10.17267/2238-2704rpf.v10i2.2971.

KLOEK, C. J. J. et al. Cost-effectiveness of a blended physiotherapy intervention compared to usual physiotherapy in patients with hip and/or knee osteoarthritis: a cluster randomized controlled trial. BMC Public Health, [s.l.], v. 18, 12p., 2018. DOI: ttps://doi.org//10.1186/s12889-018-5975-7.

LEE, A. C. COVID-19 and the Advancement of Digital Physical Therapist Practice and Telehealth. Phys Ther, [s.l.], v. 100, n. 8, p. 1.054-1.057, 2020. DOI: https://doi.org/: 10.1093/ptj/pzaa079.

NELSON, M. et al. Telerehabilitation is non-inferior to usual care following total hip replacement - a randomized controlled non-inferiority trial. Physiotherapy, [s.l.], v. 107, p. 19-27, 2020. DOI: https:// doi.org/10.1016/j.physio.2019.06.006.

NIC - NÚCLEO DE INFORMAÇÃO E COORDENAÇÃO DO PONTO BR (NIC.br); CETIC CENTRO REGIONAL DE ESTUDOS PARA O DESENVOLVIMENTO DA SOCIEDADE DA INFORMAÇÃO (Cetic.br). Painel TIC COVID-19: Pesquisa sobre o uso da Internet no Brasil durante a pandemia do novo coronavírus. 3. ed. Ensino remoto e teletrabalho, 2020. Disponível em: https://cetic.br/media/docs/publicacoes/2/20201104182616/painel_tic_covid19_3edicao_livro\%20 eletr\%C3\%B4nico.pdf. Acesso em: 15 ago. 2021.

OLIVEIRA, J. A. D. et al. Longevidade e custo da assistência: o desafio de um plano de saúde de autogestão. Cien Saúde Colet, Rio de Janeiro, v. 25, n. 10, p. 4.045-4.054, 2020. DOI: http:// dx.doi.org/10.1590/1413-812320202510.15562018.

\section{OMPI - ORGANIZAÇÃO MUNDIAL DA PROPRIEDADE INTELECTUAL. Classificação}

Internacional de Patentes. 7. ed. 1999. v. 10. Disponível em: https://www.ipdec.org/themes/ipdec/ material-de-apoio/classificacao-de-patentes.pdf. Acesso em: 20 ago. 2021.

PASTORA-BERNAL, J. M. et al. Telerehabilitation after arthroscopic subacromial decompression is effective and not inferior to standard practice: Preliminary results. J Telemed Telecare, [s.l.], v. 24, n. 6, 6p., 2018. DOI: https://doi.org/10.1177/1357633X17706583.

PAUL, L. et al. Web-based physiotherapy for people affected by multiple sclerosis: a single blind, randomized controlled feasibility study. Clin. rehabil., [s.l.], v. 33, n. 3, 12p., 2018. DOI: https://doi. org/10.1177/0269215518817080.

PIRES, E. A.; ANDRADE, J. J. S.; LORA, F. A. Mapeamento de Patentes de Respiradores. Cadernos de Prospecção, Salvador, v. 14, n. 3, p. 678-696, 2021.

ROTHGANGEL, A. et al. Traditional and augmented reality mirror therapy for patients with chronic phantom limb pain (PACT study): results of a three-group, multicentre single-blind randomized controlled trial. Clin. rehabil., [s.l.], v. 32, n. 12, 18p., 2018. DOI: https://doi. org/10.1177/0269215518785948.

SANTOS, M. T. N. et al. Aplicação da telessaúde na reabilitação de crianças e adolescentes.

Rev Paul Pediatr, [s.l.], v. 32, n. 1, p. 136-43, 2014. DOI: https://doi.org/10.1590/S010305822014000100020.

SANTOS, M. S. V. et al. Prospecção tecnológica em bases de patentes com foco em corantes e pigmentos alimentícios obtidos de fontes naturais. Res., Soc. Dev., [s.l.], v. 10, n. 3, e53810313603, 2021. DOI: http://dx.doi.org/10.33448/rsd-v10i3.13603. 
SEDETEC. Ferramenta de busca - Orbit R/etrieved. 2014. Disponível em: https://www.ufrgs.br/ sedetec/?p=660. Acesso em: 10 ago. 2021.

SHIWA, S. et al. PEDro: a base de dados de evidências em fisioterapia. Fisioter. Mov. Curitiba, v. 24, n. 3, p. 523-33, 2011. DOI: https://doi.org/10.1590/S0103-51502011000300017.

SILVA, A. B.; MENDONÇA, G. C. A Indústria de Telessaúde como uma Oportunidade para o Desenvolvimento Econômico na Saúde Pública Brasileira. Cadernos de Prospecção, Salvador, v. 12, n. 5, p. 1.459-1.473, 2019. DOI: https://doi.org/10.9771/cp.v12i5\%20Especial.32216.

TAKEMOTO, M. et al. Diet and Activity Assessments and Interventions Using Technology in Older Adults. Am J Prev Med., [s.l.], v. 55, n. 4, p. e105-e115, 2018. DOI: https://doi.org/10.1016/j. amepre.2018.06.005.

TOUSIGNANT, M. et al. A randomized controlled trial of home telerehabilitation for post-knee arthroplasty.J Telemed Telecare, [s.l.], v. 17, n. 4, p. 195-198, 2011. DOI: https://doi.org/10.1258/ jtt.2010.100602.

VAN EGMOND, M. A. Physiotherapy With Telerehabilitation in Patients With Complicated Postoperative Recovery After Esophageal Cancer Surgery: Feasibility Study. J. Med. Internet Res., [s.l.], v. 22, n. 6, 12p., 2020. https://doi.org/doi: 10.2196/16056.

\section{Sobre os Autores}

\section{Marinêz Boeing Ruaro}

E-mail:mbruaro@gmail.com ORCID: https://orcid.org/0000-0002-6300-7678

Especialista em Terapia Manual e Postura pelo Centro de Ensino Superior de Maringá (UNICESUMAR) em 2006. Endereço profissional: Universidade Estadual do Centro-Oeste (UNICENTRO), Programa de Pós-Graduação em Propriedade Intelectual e Transferência de Tecnologia (PROFNIT), Alameda Élio Antonio Dalla Vecchia, n. 838, Bairro, Vila Carli, Guarapuava, PR. CEP: 85040-167.

\section{João Afonso Ruaro}

E-mail: joaoruaro@gmail.com

ORCID: https://orcid.org/0000-0001-8323-3704

Doutor em Ciências da Saúde pela Universidade Federal do Rio Grande do Norte (UFRN) em 2014.

Endereço profissional: Universidade Estadual do Centro-Oeste (UNICENTRO), Departamento de Fisioterapia (DEFISIO/G), Setor de Ciências da Saúde, Alameda Élio Antonio Dalla Vecchia, n. 838, Bairro, Vila Carli, Guarapuava, PR. CEP: 85040-167.

\section{Daniel de Paula}

E-mail: ddepaula@unicentro.br

ORCID: https://orcid.org/0000-0001-6464-4524

Doutor em Ciências Farmacêuticas pela Faculdade de Ciências Farmacêuticas de Ribeirão Preto (FCFRP/USP) em 2007.

Endereço profissional: Universidade Estadual do Centro-Oeste (UNICENTRO), Departamento de Farmácia (DEFAR/G), Setor de Ciências da Saúde, Alameda Élio Antonio Dalla Vecchia, n. 838, Bairro, Vila Carli, Guarapuava, PR. CEP: 85040-167. 